\title{
Anti-inflammatory effects of carboxyamidotriazole on carrageenan-induced acute inflammation
}

\author{
Lei Zhu, Juan Li, Caiying Ye
}

Department of Pharmacology, Institute of Basic Medical Sciences, Chinese Academy of Medical Sciences \& School of Basic Medicine, Peking Union Medical College, China

OBJECTIVE: To investigate the therapeutical effects of carboxyamidotriazole (CAI) in a rat model of carrageenaninduced paw acute inflammation and its mechanisms. METHODS: Wistar rats were randomly divided into normal group, two vehicle groups (Polyethylene glycol 400 control and normal sodium control group), CAI-treated groups (10, 20, 30 $\mathrm{mg} / \mathrm{kg}$ ) and positive control dexamethasone group $(0.8 \mathrm{mg} / \mathrm{kg})$. After the drugs were administered for 3 days, the paw acute inflammation was induced by carrageenan except normal group. The paw swelling was measured using a plethysmometer. Myeloperoxidase (MPO) activity and nitric oxide (NO) content were determined by spectrophotometric method, and the contents of PGE2, TNF-alpha, IL-1beta, IL-10 and cytokine-induced neutrophil chemoattractant 1 (CNCI-1) were measured by ELISA. The inducible nitric oxide synthase (iNOS) expression was detected by Western blot method. RESULTS: The treatment of CAI inhibited the carrageenan-induced paw swelling. Meanwhile, CAI decreased the up-regulated MPO, NO, PGE2,TNF-alpha, IL-1beta, and CNCI-1 in the inflamed paw tissue, and increased IL-10 levels. CAI also reduced the protein expression of iNOS. CONCLUSION: CAI has remarkable inhibitory effects on carrageenan-induced paw acute inflammation in the rats, and its mechanisms may be related to decreasing the inflammatory mediators and reducing the neutrophils infiltration. 